\title{
EL CONFLICTO DE ROL DE GÉNERO MASCULINO Y SU VINCULACIÓN CON EL ACOSO ESCOLAR (BULLYING)
}

\author{
RAÚl NAVARRO \\ ELISA LARRAÑAGA \\ SANTIAGO YUBERO \\ Universidad de Castilla-La Mancha
}

Recibido: 4-03-2015

Aceptado: 28-04-2015

\section{Resumen}

En este estudio se analiza la asociación entre el conflicto de rol de género masculino y las conductas de acoso y victimización escolar. La muestra estuvo formada por 786 chicos estudiantes españoles, de edades comprendidas entre los 11 y los 17 años $(\mathrm{M}=14.5, \mathrm{DT}=1.58)$. Se establecieron tres grupos de experimentación del conflicto de rol de género tanto a nivel global como en cada uno de sus patrones: nivel alto, medio y bajo. Los resultados de los distintos análisis de varianza indicaron que el constructo de conflicto de rol de género y los patrones de conflicto que lo componen está relacionados con las distintas formas de acoso y victimización escolar. El acoso escolar parece configurarse como la respuesta a la inadecuación a los roles de género restrictivos y también una forma de penalizar aquellos que no se adhieren a ellos.

Palabras clave: conflicto de rol de género masculino, acoso escolar, adolescencia.

\begin{abstract}
The aim of this study was to analyze the association between the masculine gender role conflict and school bullying. The sample was made up of 786 Spanish adolescents boys, aged 12 to 17 years $(\mathrm{M}=14.5, \mathrm{SD}=1.58)$. Three groups of adolescents boys were distinguished according with their experimentation of the gender role conflict: high, average and low. The results of the ANOVAS indicated that the construct of gender role conflict and their conflict patterns were related to the different forms of
\end{abstract}


school bullying and victimization. Bullying seems to be configured as a response to the fail in adhering the restrictive gender roles and also a way of punish peers who do not adhere these restrictive roles.

Key-words: male gender role conflict, school bullying, adolescence.

Feminismo/s 25, junio 2015, pp. 89-110 


\section{Introducción}

Diversas investigaciones han mostrado que los chicos, comparados con las chicas, se encuentran más frecuentemente implicados en el bullying escolar tanto en el rol de agresor como en el de víctima ${ }^{1}$. Esta mayor implicación de los chicos se mantiene incluso cuando se analizan conductas de tipo indirecto o relacional que han sido tradicionalmente asociadas con las chicas ${ }^{2}$. Entre los factores que se han relacionado con la mayor implicación de los chicos se encuentra la construcción del propio autoconcepto de rol de género. Concretamente, la interiorización de rasgos de género como resultado de los procesos de socialización que marcan diferencias entre chicos y chicas en su orientación hacia objetivos más individualistas, el deseo por obtener posiciones de poder entre los grupos de iguales o la agresividad como componente de la identidad ${ }^{3}$. En este sentido, la investigación previa ha señalado que los rasgos estereotípicos masculinos están asociados con una mayor tendencia a comportarse de forma agresiva o verse implicado como agresor en las dinámicas de bullying ${ }^{4}$.

Actualmente, los estudios empíricos no sólo analizan la medida en que la conformidad con los ideales masculinos predispone a los chicos a comportarse de forma agresiva. La investigación psicológica trata ahora de conocer en qué medida la conducta agresiva puede ser el producto del estrés y el conflicto

1. CARD, Noel A., et al. «Direct and indirect aggression during childhood and adolescence: A meta-analytic review of gender differences, intercorrelations, and relations to maladjustment». Child development 79 (2008), pp. 1185-1229.

2. ARTZ, Sibylle; WASSILIS Kassis y MOLDENHAUER, Stephanie. «Rethinking indirect aggression: the end of the mean girl myth». Victims \& Offenders 8 (2013), pp. 308-328.

3. WiTT, Susan D. «The influence of peers on children's socialization to gender roles». Early Child Development and Care 162 (2000), pp. 1-7.

4. GINI, Gianluca, y Pozzoli, Tiziana. «The role of masculinity in children's bullying». Sex Roles 54 (2006), pp. 585-588.

NAVARRO, Raúl, LARRAÑAGA, Elisa y YUBERO, Santiago. «Bullying-victimization problems and aggressive tendencies in Spanish secondary schools students: the role of gender stereotypical traits». Social Psychology of Education 14 (2011), pp. 457-473.

IDEM. «Gender Identity, Gender-Typed Personality Traits and School Bullying: Victims, Bullies and Bully-Victims». Child Indicators Research (2015), pp.1-20. 
que jóvenes y adultos pueden experimentar al tratar de adherirse a dichas expectativas del rol de género ${ }^{5}$. Tratar de adherirse a los estándares culturales masculinos puede provocar un conflicto de naturaleza intra e interpersonal por el que el individuo siente que su conducta, sus actitudes y su intereses no se ajustan a sus propias creencias de género, causándole malestar 6 . Un conflicto masculino conocido como conflicto de rol de género (Gender Role Conflict, GRC) que podría encontrarse tras algunas manifestaciones de violencia de las que los hombres participan.

\subsection{Conflicto de rol de género}

El conflicto de rol de género es definido como el estado psicológico en que los roles de género socializados producen consecuencias negativas sobre el individuo o sobre los otros. Aparece cuando los roles de género rígidos, sexistas o restrictivos provocan una limitación personal, una devaluación o desvalorización de uno mismo o de los otros ${ }^{7}$. El conflicto se experimenta de forma directa o indirecta en diferentes contextos cuando el individuo: 1) se desvía o incumple las normas de género o el código contenido en las nociones de masculinidad; 2) tratando de alcanzar o fallando en alcanzar las normas contenidas en los roles de género; 3 ) experimentado discrepancias entre su auto-concepto real y el auto-concepto ideal basado en los estereotipos de rol de género. La naturaleza del conflicto difiere de acuerdo al agente o agentes en conflicto, pudiendo experimentarse de tres maneras: a) cuando el individuo devalúa, restringe o vulnera su potencial por no conseguir adecuarse a las normas de rol de género; b) cuando experimenta una devaluación o restricción provocada por otros al adaptarse o desviarse de las normas de género; c) cuando devalúa o restringe a aquellos que se desvían o adaptan a las normas de género ${ }^{8}$.

5. JAKUPCAK, Matthew; LISAK, David y ROEMER, Lizabeth. «The role of masculine ideology and masculine gender role stress in men's perpetration of relationship violence». Psychology of Men \& Masculinity 3 (2002), pp. 97-106.

COHN, Amy y ZEICHNER, Amos. «Effects of masculine identity and gender role stress on aggression in men». Psychology of Men \& Masculinity 7 (2006), pp. 179-190.

6. O'NeIL, James M. «Assessing men's gender role conflict». En D. Moore y F. Leafgren (Eds), Problem solving strategies and interventions for men in conflict, Alexandria, VA, American Association for Counseling, 1990, pp. 23-38.

7. O'NeIL, James M., et al. «Gender-role conflict scale: College men's fear of femininity». Sex Roles 14 (1986), pp. 335-350.

8. GOoD, Glenn E., et al. «Male gender role conflict: Psychometric issues and relations to psychological distress». Journal of Counseling Psychology 42 (1995), pp.3-10. 
La investigación ha corroborado la existencia de este conflicto y ha informado de que está compuesto por cuatro patrones de conflicto llamados Emotividad Restringida (ER), Comportamiento afectivo limitado hacia otros hombres (CALH), Necesidad de Éxito/ Poder/Logro (NEPL), Conflicto entre las relaciones familiar y el trabajo (CRFT). El primer patrón de conflicto (ER) enfatiza la dificultad y los temores para expresar las emociones personales. El segundo patrón (CALH) implica restricciones para expresar las emociones e ideas personales a personas del mismo sexo. El tercer patrón (NEPC) involucra un temor persistente sobre el propio éxito, la obtención de poder y el estatus social en comparación con otros. El último patrón de conflicto (CRFT) refleja las dificultades para equilibrar el trabajo con las relaciones familiares lo que provoca una disminución del tiempo de ocio y relax, así como problemas de salud como el estrés 9 .

A lo largo de estos años, este constructo ha sido apoyado empíricamente por un amplio número de investigaciones en las que se ha relacionado con diversas creencias, actitudes y conductas no adaptativas. A nivel individual, el conflicto de rol de género ha sido relacionado con la depresión ${ }^{10}$, la ansiedad y el estrés ${ }^{11}$, un pobre bienestar psicológico ${ }^{12}$ y una baja autoestima ${ }^{13}$. Adicionalmente, se ha encontrado una relación positiva del conflicto de rol de género con la alexitimia ${ }^{14}$, y el abuso de sustancias y el consumo de alcohol ${ }^{15}$. A nivel interpersonal, se ha informado de una vinculación con la falta de competencia social, la timidez y la inexpresividad emocional ${ }^{16}$, problemas

9. O'NEIL, James M. «Summarizing 25 years of research on men's gender role conflict using the gender role conflict scale new research paradigms and clinical implications». The Counseling Psychologist 36 (2008), pp. 358-445.

10. Good, Glenn E. y Mintz, Laurie B. «Gender role conflict and depression in college men: Evidence for compounded risk». Journal of Counseling \& Development 69 (1990), pp. 17-21.

11. SharPe, Mark J.; HePPNER, Paul y WAYNE, Dixon. «Gender role conflict, instrumentality, expressiveness, and well-being in adult men». Sex Roles 33 (1995), pp. 1-18.

12. ÍDEM, ibídem.

13. MAHALIK, James R. et al. "A cross-national and cross-sectional comparison of men's gender role conflict and its relationship to social intimacy and self-esteem». Sex Roles 45 (2001), pp.1-14.

14. BERGER, Jill M. et al. «Impact of Gender Role Conflict, Traditional Masculinity Ideology, Alexithymia, and Age on Men's Attitudes toward Psychological Help Seeking». Psychology of Men E Masculinity 6 (2005), pp. 73-78.

15. Monk, Debra y Ricciardelli, L. A. "Three dimensions of the male gender role as correlates of alcohol and cannabis involvement in young Australian men». Psychology of Men \& Masculinity 4 (2003), pp. 57-69.

16. BRUCH, Monroe A. et al. "Shyness, masculine ideology, physical attractiveness, and emotional inexpressiveness: Testing a mediational model of men's interpersonal competence». Journal of Counseling Psychology 45 (1998), pp. 84-97. 
de unión, separación e independencia conflictiva con la familia ${ }^{17}$, mal funcionamiento y falta de satisfacción en la pareja ${ }^{18}$, y la falta de intimidad, comunicación y conexión con otros hombres ${ }^{19}$. Aunque una gran parte de la investigación sobre esta variable ha examinado los componentes del rol de género masculino y las consecuencias de estos sobre el bienestar psicológico de los hombres. Sin embargo, puesto que las mujeres, al igual que los hombres, poseen o manifiestan un amplio rango de rasgos estereotipados y creencias hacia los roles de género, también experimentan el conflicto de rol de género. De hecho, estudios previos han demostrado que su experimentación tiene efectos similares sobre el bienestar psicológico de las mujeres ${ }^{20}$. En este sentido, es necesario conceptualizar este constructo como una problemática de ambos sexos. No obstante, dado que la implicación en el bullying es mayor entre los chicos, en este estudio nos centramos en ellos asumiendo que la investigación futura deberá analizar la influencia del conflicto sobre la conducta agresiva de las chicas.

La mayoría de estudios se han conducido con poblaciones adultas, especialmente con estudiantes universitarios. Esto ha provocado que no se conozca cómo el conflicto de rol de género se manifiesta en diferentes grupos de edad. Concretamente, se conoce poco sobre cómo los adolescentes experimentan el conflicto de rol de género y cómo éste afecta a su bienestar y a sus relaciones interpersonales ${ }^{21}$. Los primeros estudios realizados con adolescentes han mostrado que el conflicto de rol de género forma parte de un proceso evolutivo que comienzan en la adolescencia y también se encuentra relacionado con el malestar psicológico, problemas familiares, conductuales y emocionales en este grupo de edad ${ }^{22}$. No obstante, la

17. BLAZINA, Christopher y WaTKINS, Edward. «Separation/individuation, parental attachment, and male gender role conflict: Attitudes toward the feminine and the fragile masculine self». Psychology of Men \& Masculinity 1 (2000), pp. 126-132.

18. ROCHLEN, Aaron B. y MAHALIK, James R. «Women's Perceptions of Male Partners' Gender Role Conflict as Predictors of Psychological Well-Being and Relationship Satisfaction». Psychology of Men \& Masculinity 5 (2004), pp. 147-157.

19. THEODORE, Harry, y LlOYD, Bibian. «Age and gender role conflict: A cross-sectional study of Australian men». Sex Roles 42 (2000), pp. 1027-1042.

20. ZAMARRIPA, Manuel, WAMPOLD, Bruce, y GREGORY, Erik. «Male gender role conflict, depression, and anxiety: clarification and generalizability to women». Journal of Counseling Psychology 50 (2003), pp. 333-338.

21. O'NEIL, James M. Op. cit.

22. Blazina, Christopher; PiseCCO, Stewart y O'Neil, James. «An Adaptation of the Gender Role Conflict Scale for Adolescents: Psychometric Issues and Correlates with Psychological Distres. Psychology of Men \& Masculinity 6 (2005), pp. 39-45.

BLAzINA, Chris, et al. «Gender role conflict scale for adolescents: Correlates with masculinity ideology». Thymos: Journal of Boyhood Studies 1 (2007), pp.191-204. 
comprensión del conflicto de rol de género en la adolescencia necesita de investigaciones que permitan generalizar estos resultados a otras culturas y conocer mejor cómo se manifiesta en diferentes grupos de edad, y su relación con otras variables que permitan conocer las consecuencias para aquellos que lo sufren ${ }^{23}$.

\subsection{Conflicto de rol de género y conducta agresiva}

Desde el conflicto de rol de género, la agresión es vista como un mecanismo de afrontamiento del estrés y malestar provocado por el intento de seguir las normas de género tradicionales, por lo que la agresión sería una forma de demostrar los propios valores de género o solucionar las amenazas a estos valores $^{24}$. La agresión se configura como un medio de proteger la identidad personal y defenderse contra los sentimientos de vulnerabilidad o inadecuación a su rol de género. En definitiva, se ha teorizado que es más probable que se produzca el uso de la agresión cuando el hombre percibe que su identidad de género es amenazada ${ }^{25}$. De esta forma, experimentar conflicto de rol de género no sólo supone una limitación a nivel personal, sino que también produce limitaciones a nivel interpersonal, en la medida en que el conflicto puede derivar en conductos de agresión o abuso, dirigidas hacia los otros ${ }^{26}$.

La mayor parte de los estudios que han relacionado la agresión interpersonal con el conflicto de rol de género han indagado sobre la violencia masculina dirigida a las mujeres dentro de las relaciones íntimas. Específicamente, los patrones de conflicto relacionados con el éxito, el poder y la competición, así como el afecto limitado hacia otros hombres, correlacionan de forma positiva con la aceptación de mitos sobre la violación ${ }^{27}$, actitudes hostiles hacia las

23. O'NeIL, James M. Op. cit.

24. Blazina, Christopher y WaTkins, Edward. Op. cit.

25. O'NeIL, James M.; GOOD, Glen y HOLMES, Sarah. «Fifteen years of theory and research on men's gender role conflict: New paradigms for empirical research». A new psychology of men (1995), pp.164-206.

O'NEIL, James M. y HARWAY, Michele. «A Multivariate Model Explaining Men's Violence Toward Women Predisposing and Triggering Hypotheses». Violence Against Women 3 (1997), pp. 182-203.

26. O'NeIL, James M. Op. cit.

27. KASSING, Leslee R.; BEESLEY, Denise y FREY, Lisa. «Gender role conflict, homophobia, age, and education as predictors of male rape myth acceptance». Journal of Mental Health Counseling 27 (2005), pp. 311-328. 
mujeres ${ }^{28}$, y actitudes positivas hacia el acoso sexual ${ }^{29}$. El conflicto vinculado al éxito, poder y competición también se ha relacionado con la agresión física dentro de las relaciones de pareja, lo que confirma que el uso de la violencia es medio para obtener poder y control sobre la víctima y afrontar así las amenazas percibidas hacia la identidad masculina ${ }^{30}$.

Los estudios que analizan la relación del conflicto de rol de género con otras formas de agresión son escasos y, hasta donde llega nuestro conocimiento, ningún estudio ha analizado la relación entre el bullying escolar y el conflicto de rol de género. El bullying, como una forma repetida de agresión física y psicológica en el contexto educativo, puede ser un modo de reafirmar la identidad masculina en situaciones en las que los adolescentes experimentan un conflicto al tratar de adherirse a los roles de género tradicionales. Igualmente, el bullying podría ser una forma de victimización entre aquellos que no se conforman a los roles de género o que muestran dificultades para adherirse a estos roles. Se trata de un aspecto que no ha sido examinado previamente $y$, sin embargo, importante si consideramos que, por ejemplo, los estudios que analizan el bullying homofóbico ${ }^{31}$ han encontrado que no todas las víctimas se identifican como homosexuales o transgénero, sino que algunos jóvenes son acosados sencillamente porque son percibidos como diferentes. Diferencia que los agresores atribuyen a su orientación sexual pero que en muchos casos está relacionada con un comportamiento de género atípico o falta de conformidad a los roles de género.

\subsection{Objetivos e hipótesis de trabajo}

Este estudio examina la asociación entre el Conflicto de Rol de Género y el bullying en chicos que cursan educación secundaria obligatoria. Aunque la asociación entre el GRC y la conducta agresiva ha sido estudiada en muestras

28. RANDO, Robert A.; Rogers, James R. y BRITTAN-Powell, Christopher S. «Gender role conflict and college men's sexually aggressive attitudes and behavior». Journal of Mental Health Counseling 20 (1998), pp. 359-369.

29. Glomb, Stephen M. y EsPelage, Dorothy L. «The Influence of Restrictive Emotionality in Men's Emotional Appraisal of Sexual Harassment: A Gender Role Interpretation». Psychology of Men E Masculinity 6 (2005), pp. 240-253.

30. SCHWARTZ, Jonathan P.; WALDO, Michael y DANIEL, David. «Gender-Role Conflict and Self-Esteem: Factors Associated With Partner Abuse in Court-Referred Men». Psychology of Men \& Masculinity 6 (2005), pp. 109-113.

31. Collier, Kate; VAn Beusekom, Gabriël; Bos, Henny y SANDFort, Theo. «Sexual orientation and gender identity/expression related peer victimization in adolescence: a systematic review of associated psychosocial and health outcomes». Journal of Sex Research 50 (2013), pp. 299-317. 
con adultos, este es uno de los primeros estudios que analiza esta asociación en una muestra de adolescentes implicados en conductas de bullying. Dado que la investigación previa ha encontrado que la experimentación del conflicto de rol de género está relacionada con el rol de agresor en poblaciones adultas, en primer lugar, hipotetizamos que el conflicto de rol de género también se relacionará con el rol de agresor entre los adolescentes, más concretamente dentro de las conductas de acoso escolar. En segundo lugar, hasta donde llega nuestro conocimiento tal y como se informa en la revisión sobre la investigación de esta variable de género ${ }^{32}$, ningún estudio ha examinado la relación del conflicto con los procesos de victimización. Sin embargo, se ha teorizado que los hombres que sufren el conflicto pueden estar expuestos a procesos de victimización al no adecuarse a los roles de género prescritos socialmente $e^{33}$. Por este motivo, también hipotetizamos que el conflicto de rol de género estará relacionado con la victimización en contexto escolar. No se realizan predicciones específicas sobre las relaciones entre los distintos patrones de conflicto y las formas de agresión y victimización escolar, al no disponer de estudios previos que analicen la asociación diferencial de los patrones con formas específicas de acoso escolar.

\section{Método}

\subsection{Muestra y procedimiento}

Los datos fueron obtenidos en 15 centros de educación secundaria obligatoria de titularidad pública elegidos al azar entre una lista proporcionada por la Consejería de Educación de la Comunidad de Castilla-La Mancha. Después de que 56 cuestionarios fueron rechazados porque estaban incompletos, el número final de participantes fue de 786 chicos con edades comprendidas entre los 12-18 años (Edad Media: 14.5 años; DT=1.58).

Los datos se obtuvieron mediante cuestionarios de auto informe. Se solicitó la participación voluntaria de los estudiantes y participaron en el estudio quienes contaban con el consentimiento escrito de sus padres. Los cuestionarios se administraron de forma anónima sin que hubiese ningún tipo de información que permitiese identificar a los participantes. Dos investigadores administraron los cuestionarios clarificando el significado de ciertos ítems o respondiendo a las preguntas de los estudiantes cuando fuera necesario. La administración en cada clase duró aproximadamente 35 minutos.

32. O'NeIL, James M. Op. cit.

33. ÍDEM, Ibídem. 


\subsection{Instrumentos}

Se describen en este apartado los dos instrumentos de medida utilizados en la investigación.

Conflicto de Rol de Género. La exploración de las consecuencias negativas de la adhesión a los roles de género tradicionales se ha realizado mediante la Escala de Conflicto de Rol de Género para Adolescentes (GRCS- $\mathrm{A}^{34}$ ). Concretamente se ha utilizado la versión española ${ }^{35}$. Esta escala está constituida por 29 enunciados en los que los adolescentes informan de su acuerdo o desacuerdo con cada enunciado en una escala tipo Likert con un rango de 1 (muy en desacuerdo) a 6 (muy de acuerdo). La estructura factorial muestra cuatro patrones de conflicto: Emotividad Restringida (RE, Restricted Emotionality) con 9 ítems, por ejemplo: «No me gusta mostrar mis emociones a otras personas»); Afecto Limitado entre Hombres (RABM, Restricted Affection Between Men) con 7 ítems, por ejemplo «Me resulta difícil abrazar a otros hombres»; Conflicto entre Trabajo, Escuela y Familia (CWSF, Conflict Between Work, School, and Family) con 7 ítems, por ejemplo «Me resulta difícil encontrar tiempo para descansar»; Necesidad de Éxito y Logro (NSA, Need for Success and Achievement) con 6 ítems, por ejemplo «Ganar dinero es parte de mi idea de lo que es ser un hombre de éxito» que es reflejo de aspectos de la ideología masculina tradicional más que del conflicto de rol de género. Cuanto mayor es la puntuación en cada una de las subescalas, mayor es el conflicto de rol de género. Los índices de fiabilidad en este estudio para cada una de las subescalas son: Necesidad de Éxito y Logro, $\alpha=.72$; Emotividad Restringida, $\alpha=.79$; Afecto Limitado entre Hombres, $\alpha=.82$; y Conflicto entre Trabajo, Escuela y Familia, $\alpha=.78$.

Acoso escolar. Para medir las conductas de acoso y victimización escolar se emplearon los ítems del «Instrument to assess the incidence of involvement in bully/victim interaction at school» ${ }^{36}$. El cuestionario está organizado en dos bloques de acuerdo a la posible situación de acoso: víctima (ser acosado), agresor (participar en el acoso a alguien). En cada uno de los dos bloques se recoge información sobre distintas formas de agresión que aparecen en

34. Blazina, Christopher, PiseCco, Stewart y O'Neil, James M. Op. cit.

35. LARRAÑAGA, Elisa, et al. «Validación de la versión española de la Gender Role Conflict Scale for Adolescents (GRCS-A)». Anales de psicología 29 (2013), pp. 549-557.

36. RigBY, Ken y BAGSHAW, Dale. «Prospects of adolescent students collaborating with teachers in addressing issues of bullying and conflict in schools». Educational Psychology 23 (2003), pp. 535-546. 
la mayoría de las descripciones y definiciones del acoso escolar: agresión física (Ejemplo: «He pegado o empujado a alguien»), y verbal (Ejemplo: «He hablado de alguien a sus espaldas o he dicho cosas falsas sobre ellos»), y exclusión social del grupo de iguales (Ejemplo: ignorar, no dejar participar en actividades). Los participantes debían responder en una escala tipo Likert de 4 puntos ( $0=$ Nunca y $3=$ Diariamente) si durante los pasados dos meses habían participado de la agresión repetida a otras personas, o habían sido víctimas de estas agresiones. La consistencia interna para la escala sobre el rol de agresor fue de.79, y de.74 para el rol de víctima.

\subsection{Estrategia de análisis}

Atendiendo a los objetivos e hipótesis en este trabajo de investigación, en primer lugar, se calcularon las puntuaciones medias de los participantes para cada uno de las variables de estudio (Tabla 1). En segundo lugar, se realizó un análisis de regresión múltiple para evaluar la contribución de los patrones de conflicto de rol de género en cada una de las formas de acoso y victimización escolar examinadas. Finalmente, se analizaron las diferencias en el acoso y victimización escolar de acuerdo a los niveles de experimentación del conflicto de rol de género. Para ello, tras comprobar mediante la prueba de Kolmogorov-Smirnov que las puntuaciones en el conflicto de rol de género se distribuían normalmente, se realizaron una serie de análisis de varianza (ANOVAs) para conocer las diferencias en el acoso y la victimización escolar de acuerdo a tres niveles diferentes de experimentación del conflicto (alto/ medio/bajo) mediante la prueba Bonferroni.

La agrupación de los participantes en los tres niveles de experimentación del conflicto se realizó considerando el percentil en que se sitúan sus puntuaciones tanto en una medida global del conflicto de rol de género como en cada uno de los patrones de conflicto identificados por la investigación previa. De esta forma, los participantes fueron clasificados considerando el percentil 25, 50 y 75 . Aquellos cuyas puntuaciones se encontraban en el percentil 25 inferior de la distribución fueron incluidos dentro del grupo con un nivel bajo de experimentación del conflicto. Los participantes cuyas puntuaciones se situaban por encima del percentil 25 superior de la distribución, fueron categorizados dentro del grupo con un nivel alto de experimentación en las variables ya descritas. Por último, los sujetos cuyas puntuaciones se encuentran por encima del percentil 25 inferior de la distribución y por debajo del percentil 25 superior de la distribución, se incluyen dentro del grupo con un nivel medio de experimentación. Este método de categorización se encuentra en la línea de la investigación previa, que ha analizado la 
relación entre la conducta agresiva y otros factores asociados a su manifestación ${ }^{37}$. Todos los datos fueron analizados utilizando el programa estadístico SPSS 19.0.

\section{Tabla 1}

Estadísticos descriptivos de las conductas de acoso, victimización escolar y conflicto de rol de género

\begin{tabular}{|l|c|c|c|c|}
\hline & Mínimo & Máximo & Media & Desv. Tip. \\
\hline Acoso físico & 0 & 3 & 0.190 & 0.32 \\
\hline Acoso Verbal & 0 & 3 & 0.433 & 0.58 \\
\hline Acoso/Exclusión & 0 & 3 & 0.195 & 0.36 \\
\hline Victimización Física & 0 & 3 & 0.139 & 0.32 \\
\hline Victimización Verbal & 0 & 3 & 0.392 & 0.58 \\
\hline Victimización/Exclusión & 0 & 3 & 0.091 & 0.36 \\
\hline Emotividad Restringida & 1 & 6 & 3.1066 & 1.01 \\
\hline Afecto limitado con otros hombres & 1 & 6 & 3.1599 & 1.2 \\
\hline Conflicto entre trabajo, escuela y familia & 1 & 6 & 3.2877 & 1.20 \\
\hline Necesidad de éxito y logro. & 1 & 6 & 3.6050 & 1.02 \\
\hline
\end{tabular}

\subsection{Resultados}

Exploración de la relación entre los constructos de género y la conducta agresiva: análisis de regresión

Con el objetivo de determinar la medida en que cada una de las variables examinadas contribuye a explicar la agresión informada por los estudiantes, se realizó un análisis de regresión múltiple para evaluar la contribución de los patrones de conflicto de rol de género, en cada una de las formas de acoso y victimización escolar. Los resultados de este análisis se incluyen en la Tabla 2 mostrando una asociación significativa entre los patrones de conflicto de rol de género y la mayoría de las conductas de acoso y victimización. Los patrones de conflicto de rol de género explican de forma significativa parte de la varianza en el acoso verbal y mediante exclusión y, también, de todas las formas de victimización examinadas.

37. KilPATRICK, Michelle y Kerres, Christine. «Perceptions of the Frequency and Importance of Social Support by Students Classified as Victims, Bullies, and Bully/Victims in an Urban Middle School». School Psychology Review 32 (2003), pp.471-489. 
Tabla 2

Análisis de regresión del conflicto de rol de género sobre las formas de acoso y victimización

\begin{tabular}{|c|c|c|c|c|c|c|}
\hline \multirow{2}{*}{} & \multicolumn{3}{|c|}{ Formas de Acoso } & \multicolumn{3}{c|}{ Formas de Victimización } \\
\cline { 2 - 7 } & \multicolumn{3}{|c|}{$\beta$} & \multicolumn{3}{c|}{$\beta$} \\
\cline { 2 - 7 } CRG & $\begin{array}{c}\text { Acoso } \\
\text { Físico }\end{array}$ & $\begin{array}{c}\text { Acoso } \\
\text { Verbal }\end{array}$ & $\begin{array}{c}\text { Acoso- } \\
\text { Exclusión }\end{array}$ & $\begin{array}{c}\text { Victimización } \\
\text { física }\end{array}$ & $\begin{array}{c}\text { Victimización } \\
\text { verbal }\end{array}$ & $\begin{array}{c}\text { Victimización } \\
\text { exclusión }\end{array}$ \\
\hline ER & .060 & -.058 & -.049 & .162 & .119 & .136 \\
\hline ALH & .014 & .072 & .052 & -.017 & .029 & -.053 \\
\hline CTEF & .077 & .184 & .107 & -.024 & .026 & .007 \\
\hline NEL & -.077 & -.009 & .008 & .056 & .024 & .028 \\
\hline$R^{2}$ & .005 & .031 & .007 & .025 & .025 & .012 \\
\hline$F$ & 1.964 & $7.076^{* * *}$ & $2.402^{*}$ & $5.823^{* * *}$ & $4.819^{* * *}$ & $3.341^{* *}$ \\
\hline
\end{tabular}

CRG: Conflicto de Rol de Género. ER: Emotividad Restringida. ALH: Afecto limitado hacia otros hombres. CTEF: Conflicto entre trabajo, escuela y familia. NEL: Necesidad de éxito y logro.

${ }^{*} \mathrm{p}<.05{ }^{* *} \mathrm{p}<.01{ }^{* * *} \mathrm{p}<.001$

Análisis del impacto del conflicto de rol de género en las conductas de acoso y victimización escolar de los estudiantes de secundaria

Confirmada la existencia de efectos significativos entre las variables de estudio, en este apartado se muestra el análisis diferencial realizado para comprobar la asociación entre diferentes niveles de experimentación del conflicto de rol de género y las conductas de acoso y victimización escolar. Los participantes fueron agrupados de acuerdo a un nivel bajo, medio o alto de conflicto de rol de género siguiendo el procedimiento previamente descrito, considerando en primer lugar una medida global del conflicto y, posteriormente, analizando sus diferentes patrones.

En la Tabla 3 se muestran los resultados relativos a la asociación del conflicto de rol de género como constructo global con las formas de acoso y victimización escolar. En relación a las formas de acoso, los chicos con un nivel alto de conflicto informan de una mayor implicación en el acoso verbal que los situados en otros niveles. El acoso mediante exclusión es menos informado por aquellos que experimentan un nivel bajo de conflicto. En la formas de victimización, las diferencias significativas se producen en la victimización física y verbal. Los chicos que experimentan un nivel alto de conflicto sufren una mayor victimización física y verbal. 


\section{Tabla 3}

ANOVA entre el conflicto de rol de género, acoso y victimización escolar y Bonferroni Post hoc ${ }^{1}$

\begin{tabular}{|l|c|c|c|c|}
\hline & \multicolumn{4}{|c|}{ Conflicto de Rol de Género } \\
\hline Acoso & Nivel Bajo & Nivel Medio & Nivel Alto & F $(2,786)$ \\
\hline Físico & 0.147 & 0.174 & 0.218 & 1.636 \\
\hline Verbal & $0.309^{\mathrm{b}}$ & $0.411^{\mathrm{b}}$ & $0.523^{\mathrm{a}}$ & $6.659^{* * *}$ \\
\hline Exclusión & $0.066^{\mathrm{a}}$ & $0.214^{\mathrm{b}}$ & $0.237^{\mathrm{b}}$ & $4.106^{* *}$ \\
\hline Victimización & \multicolumn{5}{|c|}{} \\
\hline Física & $0.114^{\mathrm{b}}$ & $0.114^{\mathrm{b}}$ & $0.187^{\mathrm{a}}$ & $6.122^{* *}$ \\
\hline Verbal & $0.300^{\mathrm{b}}$ & $0.348^{\mathrm{b}}$ & $0.489^{\mathrm{a}}$ & $4.558^{* * *}$ \\
\hline Exclusión & 0.047 & 0.082 & 0.124 & 1.916 \\
\hline
\end{tabular}

${ }^{1} \alpha=.05 ; \mathrm{a}>\mathrm{b}>\mathrm{c}$

${ }^{*} \mathrm{p}<.05{ }^{* *} \mathrm{p}<.01{ }^{* * *} \mathrm{p}<.001$

Posteriormente se analizó la asociación entre los diferentes patrones de conflicto y el acoso y la victimización escolar (Tablas 4 y 5). Los resultados muestran que los chicos que experimentan un nivel alto de «emotividad restringida» están más involucrados en el acoso físico a otros compañeros. En cuanto a las formas de victimización, los chicos con un alto nivel de «emotividad restringida» sufren mayores índices de victimización física, verbal y mediante exclusión (Tabla 4). No se encontró ninguna asociación significativa entre el patrón de «afecto limitado hacia otros hombres» y las formas de acoso y victimización escolar. 


\section{Tabla 4}

ANOVA entre los patrones de «emotividad restringida», «afecto limitado hacia otros hombres», acoso y victimización escolar y Bonferroni Post hoc ${ }^{1}$

\begin{tabular}{|l|c|c|c|c|c|c|c|c|}
\hline & \multicolumn{9}{|c|}{ Emotividad Restringida } & \multicolumn{3}{c|}{$\begin{array}{c}\text { Afecto limitado hacia otros } \\
\text { hombres }\end{array}$} \\
\hline Acoso & $\begin{array}{c}\text { Nivel } \\
\text { Bajo }\end{array}$ & $\begin{array}{c}\text { Nivel } \\
\text { Medio }\end{array}$ & $\begin{array}{c}\text { Nivel } \\
\text { Alto }\end{array}$ & $\begin{array}{c}\text { F } \\
(2,762)\end{array}$ & $\begin{array}{c}\text { Nivel } \\
\text { Bajo }\end{array}$ & $\begin{array}{c}\text { Nivel } \\
\text { Medio }\end{array}$ & $\begin{array}{c}\text { Nivel } \\
\text { Alto }\end{array}$ & $\begin{array}{c}\text { F } \\
(2,768)\end{array}$ \\
\hline Físico & $0.113^{\mathrm{b}}$ & $0.167^{\mathrm{b}}$ & $0.243^{\mathrm{a}}$ & $3.366^{*}$ & 0.188 & 0.175 & 0.201 & 0.413 \\
\hline Verbal & 0.369 & 0.442 & 0.460 & 1.838 & 0.358 & 0.419 & 0.465 & 1.186 \\
\hline Exclusión & 0.156 & 0.174 & 0.228 & 1.092 & 0.226 & 0.154 & 0.242 & 2.542 \\
\hline Victimización \\
\hline Física & $0.100^{\mathrm{b}}$ & $0.107^{\mathrm{b}}$ & $0.217^{\mathrm{a}}$ & $10.157^{* * *}$ & 0.122 & 0.118 & 0.168 & 2.291 \\
\hline Verbal & $0.278^{\mathrm{b}}$ & $0.356^{\mathrm{b}}$ & $0.504^{\mathrm{a}}$ & $7.358^{* * *}$ & 0.452 & 0.343 & 0.438 & 2.597 \\
\hline Exclusión & $0.034^{\mathrm{b}}$ & $0.070^{\mathrm{b}}$ & $0.154^{\mathrm{a}}$ & $5.629^{* * *}$ & 0.113 & 0.089 & 0.091 & 0.095 \\
\hline
\end{tabular}

${ }^{1} \alpha=.05 ; \mathrm{a}>\mathrm{b}>\mathrm{c}$

${ }^{*} \mathrm{p}<.05 * * \mathrm{p}<.01 * * * \mathrm{p}<.001$

El patrón de «conflicto entre trabajo, familia y escuela» se encuentra asociado con el acoso verbal y mediante exclusión (Tabla 5). Específicamente, la implicación en el acoso verbal es mayor a medida que el nivel de conflicto experimentado es más elevado. Las conductas de exclusión son más protagonizadas por los chicos que experimentan un nivel de conflicto medio o alto. 


\section{Tabla 5}

ANOVA entre los patrones de «conflicto entre trabajo, escuela y familia», «necesidad de éxito y logro», acoso y victimización escolar y Bonferroni Post hoc $^{1}$

\begin{tabular}{|c|c|c|c|c|c|c|c|c|}
\hline \multirow{2}{*}{\begin{tabular}{|l} 
\\
Acoso
\end{tabular}} & \multicolumn{4}{|c|}{$\begin{array}{l}\text { Conflicto entre trabajo, escuela y } \\
\text { familia }\end{array}$} & \multicolumn{4}{|c|}{ Necesidad de éxito y logro } \\
\hline & $\begin{array}{l}\text { Nivel } \\
\text { Bajo }\end{array}$ & $\begin{array}{l}\text { Nivel } \\
\text { Medio }\end{array}$ & $\begin{array}{l}\text { Nivel } \\
\text { Alto }\end{array}$ & $\begin{array}{c}F \\
(2,776)\end{array}$ & $\begin{array}{l}\text { Nivel } \\
\text { Bajo }\end{array}$ & $\begin{array}{l}\text { Nivel } \\
\text { Medio }\end{array}$ & $\begin{array}{c}\text { Nivel } \\
\text { Alto }\end{array}$ & $\begin{array}{c}\mathrm{F} \\
(2,776)\end{array}$ \\
\hline Físico & 0.161 & 0.184 & 0.209 & 0.694 & 0.070 & 0.089 & 0.106 & 1.344 \\
\hline Verbal & $0.286^{c}$ & $0.407^{\mathrm{b}}$ & $0.540^{\mathrm{a}}$ & $10.584 * * *$ & 0.111 & 0.212 & 0.218 & $4.504 * *$ \\
\hline Exclusión & $0.080^{\mathrm{b}}$ & $0.206^{\mathrm{a}}$ & $0.243^{\mathrm{a}}$ & $4.446^{* *}$ & 0.194 & 0.192 & 0.188 & 2.117 \\
\hline \multicolumn{9}{|c|}{ Victimización } \\
\hline Física & 0.161 & 0.112 & 0.167 & 2.633 & $0.115^{\mathrm{b}}$ & $0.120^{\mathrm{b}}$ & $0.188^{\mathrm{a}}$ & $3.970^{*}$ \\
\hline Verbal & 0.352 & 0.366 & 0.449 & 1.927 & $0.376^{\mathrm{b}}$ & $0.326^{\mathrm{b}}$ & $0.511^{\mathrm{a}}$ & $7.730 * * *$ \\
\hline Exclusión & 0.080 & 0.070 & 0.127 & 1.949 & 0.067 & 0.084 & 0.119 & 1.059 \\
\hline
\end{tabular}

${ }^{1} \alpha=.05 ; \mathrm{a}>\mathrm{b}>\mathrm{c}$

${ }^{*} \mathrm{p}<.05{ }^{* *} \mathrm{p}<.01{ }^{* * *} \mathrm{p}<.001$

Analizado el patrón de «necesidad de éxito y logro», aparecen diferencias en el acoso verbal perpetrado por los chicos (Tabla 5). Aquellos con un nivel bajo de conflicto se encuentran menos implicados en el acoso verbal. En cuanto a las formas de victimización, la victimización física sufrida por los chicos es mayor entre los que experimentan un elevado patrón de conflicto. De igual manera, un alto nivel de «necesidad de éxito y logro» está asociado con una mayor victimización verbal.

\section{Discusión}

Los datos obtenidos confirman el efecto diferencial del constructo de conflicto de rol de género y los patrones de conflicto que lo componen sobre las formas de acoso y victimización escolar. En relación con el rol de agresor, los resultados muestran que los chicos que están más implicados en el acoso físico experimentan una mayor «emotividad restringida». La dificultad para expresar las propias emociones y sentimientos puede conducir a un estilo de interacción hostil hacia los otros, que desemboque en la agresión, tal y como 
han confirmado otras investigaciones ${ }^{38}$. En el acoso verbal, los chicos que informan de su implicación también lo hacen de un mayor «conflicto entre trabajo, escuela y familia» y «emotividad restringida». Las dificultades para equilibrar el trabajo escolar con las relaciones familiares puede provocar un mayor estrés, lo que unido al temor persistente sobre el propio éxito puede hacerles más proclives a la implicación en el acoso como una forma de alcanzar mayor poder y estatus, así como liberar la tensión que les produce la limitación de su tiempo libre. El «conflicto entre trabajo, escuela y familia» se asocia de forma significativa con el acoso mediante exclusión. Los resultados confirman que, el acoso escolar puede constituir un medio para proteger su identidad personal y defenderse de sus sentimientos de vulnerabilidad e inadecuación a las normas de género ${ }^{39}$.

Se confirman así los resultados de la investigación previa que relacionaban el conflicto de rol de género y, también, sus diferentes patrones con la conducta agresiva $^{40}$, en este caso entre adolescentes y dentro del marco del acoso escolar. Aquellos que sufren el conflicto o experimentan alguno de sus patrones sufren una devaluación en su autoconcepto de género que pueden llevarle a actuar de forma agresiva como medio de afrontamiento del estrés y malestar provocado por el intento de seguir las normas de género. Es posible que aquellos participantes que experimenten el conflicto perciban la agresión como un modo de defender su adecuación a las normas de género tradicionales o responder a la amenaza de estas normas y, por tanto, se orienten más hacia la agresión ${ }^{41}$. No obstante resulta llamativo que, con la excepción del patrón emotividad restringida, el acoso físico no se encuentra relacionado con el conflicto de rol de género. La investigación previa ha indicado que se encuentra relacionado con actitudes favorables hacia la agresión física y, también con la tendencia a agredir físicamente al menos en contexto experimentales ${ }^{42}$. Parece, por tanto, que el conflicto de rol de género, más que un desencadenante directo de la agresión física, actúa como factor de riesgo y sea el contexto el que facilite o mitigue esta respuesta. En cualquier caso, se necesitan estudios que analicen esta cuestión más detenidamente y de una forma longitudinal.

38. HAYES, Jeffrey A. y MAHALIK, James R. «Gender role conflict and psychological distress in male counseling center clients». Psychology of Men E Masculinity 1 (2000), pp. 116-125.

39. Blazina, Christopher y Watkins, Edward. Op. cit.

40. CoHn, Amy y Zeichner, Amos. Op. cit.

41. O'NeIL, James M. y HARWAY, Michele. Op. cit.

42. Cohn, Amy M.; ZeiCHner, Amos y SEIBERT, Alana. «Labile affect as a risk factor for aggressive behavior in men». Psychology of Men E Masculinity 9 (2008), pp. 29-39. 
La relación del conflicto de rol de género con las formas de victimización es más fuerte y significativa, que en el caso de las formas de acoso. La «emotividad restringida» se encuentra asociada de forma significativa a la victimización física, verbal y mediante exclusión sufrida por los chicos. Igualmente, la «necesidad de éxito y logro» influye sobre la victimización física y verbal. Si tenemos en cuenta que el conflicto de rol de género está originado por la falta de adecuación a los rasgos y roles de género tradicionales relacionados con la expresión de sentimientos, las relaciones con el propio sexo o la búsqueda de éxito, la experimentación de dificultades para expresar las emociones y necesidades personales, así como un deseo de obtener un mayor poder y estatus, debemos considerar estos patrones como factores de riesgo para la victimización escolar. El bullying podría tener como objetivo la conformidad a las normas de género o la construcción de la víctima como un competidor en la lucha por el poder y el estatus, ya que aquellos que sufren el conflicto experimentan temor por su propio éxito. Además, el acoso se vería facilitado por las dificultades de quienes sufren el conflicto para comunicar sus emociones y problemas, así como un posible aislamiento social al experimentar dificultades para conciliar su vida escolar y familiar. La victimización también puede ser la expresión de un prejuicio y la discriminación hacia aquellos que no se conforman a las normas de género tradicionales, no sienten que su género se corresponda con su sexo biológico o que muestran interés sexual por personas del mismo sexo. Un tipo de bullying que, según la investigación previa, tienen consecuencias más graves para quien lo sufre que otras formas de bullying si esta base discriminatoria ${ }^{43}$. Los resultados encontrados contribuyen a ampliar el conocimiento de cómo el conflicto de rol de género influye sobre la victimización sufrida por aquéllos que experimentan el conflicto de rol de género ${ }^{44}$.

En cualquier caso, los resultados de esta investigación confirman que el acoso escolar es el producto de una compleja combinación de variables, entre las que debe considerarse el género $y$, concretamente, el conflicto de rol de género. A pesar de que la investigación es todavía incipiente y de que se necesita un análisis más profundo de la relación entre la conducta agresiva y la construcción de las variables de género, los resultados encontrados deben llevarnos hacia una reflexión sobre los valores y conductas que desde los procesos de socialización de género se trasmiten a los chicos y que los posicionan

43. Russell, Stephen; Sinclair, Katerina; Poteat, Paul y Koening, Brian. «Adolescent health and harassment based on discriminatory bias». American Journal of public health 102 (2012), pp. 493-495.

44. O'NEIL, James M. Op. cit. 
ante una situación de vulnerabilidad a la hora de sufrir o ejercer la agresión dentro del acoso escolar. La información obtenida puede ser utilizada en la mejora de la efectividad de los programas de prevención e intervención sobre el acoso y la victimización.

A tenor de los resultados encontrados, se puede realizar una serie de recomendaciones encaminadas a la mejora de los programas de prevención e intervención. Puesto que las experiencias escolares son parte significativa de lo que los estudiantes aprenden de los otros, es necesario intervenir sobre aquello que representa una influencia negativa, sobre todo si se tiene en cuenta que los problemas de acoso entre iguales pueden ser la antesala de otras conductas problemáticas posteriores: abuso sexual, violencia en las relaciones de pareja o delincuencia ${ }^{45}$.

En este sentido, los resultados de esta investigación señalan la importancia de adoptar una perspectiva de género en la prevención sobre el acoso escolar, en la que, por un lado, se analicen las características y complejidad de las relaciones de género que se producen en los centros escolares, lo que implica analizar detenidamente las dinámicas de acoso y victimización escolar atendiendo a la influencia de creencias culturales vinculadas al género, tal y como se ha mostrado en esta investigación. Por otro lado, se estimule a que profesores y padres razonen y confronten sus propias actitudes y experiencias relacionadas con el género y la agresión, lo que obliga a que sean cuestionadas las creencias de género que se están promoviendo en los centros con el objetivo de proporcionar un amplio rango de iniciativas en las que tanto chicos como chicas puedan experimentar representaciones de género no tradicionales. Y, por último, se facilite una mejora de las relaciones entre los géneros a través de actividades que permitan a los estudiantes explorar sus diferencias, hablar sobre sus ideas acerca del acoso entre iguales, implicarlos en el desarrollo de medidas para su prevención, y facilitar espacios que promuevan la amistad y cooperación entre personas con características diversas: edad, procedencia y género.

El acoso entre iguales puede estar vinculado a la negociación de la identidad de género de algunos de sus participantes tal y como apuntan los resultados encontrados. Las trasgresiones de las diferentes barreras del comportamiento de género aceptado para chicos pueden ser castigadas a través de la presión de los iguales. Los chicos que no se comportan de acuerdo a las normas dominantes de masculinidad, pueden sufrir acoso (bullying homofóbico).

45. COCKER, Ann L. et al. «Dating violence victimization and perpetration rates among high school students». Violence against women 20 (2014), pp. 1220-1238. 
En consecuencia, la intervención debería tender a la mejora de habilidades de relación intra-género e inter-género, acabar con la idea de que es necesaria la agresión para alcanzar una posición de poder dentro de las relaciones personales, romper con la normalizada asociación de lo masculino con la violencia y promover relaciones positivas basadas en la aceptación de las diferencias. Advirtiendo que el acoso entre iguales puede ser una reacción a la diferencia y un intento de buscar la conformidad a las normas de género de los que son construidos como «diferentes», será necesario promover valores relacionados con la aceptación de la diversidad.

Para terminar, es importante señalar que, aunque los resultados expuestos son significativos, deben ser analizados con cautela considerando que la muestra sobre la que se han realizado los cálculos estadísticos es reducida y se circunscribe a centros de secundaria de una única comunidad. Las asociaciones encontradas entre el conflicto de rol de género y las formas de acoso escolar pueden ser diferentes en otras muestras. La investigación futura debería replicar este estudio con una muestra más representativa. Además, es necesario analizar cómo el conflicto de rol de género experimentando por las chicas se relaciona con las formas de acoso y victimización. Igualmente, la interpretación de los resultados debe realizarse con cautela debido la naturaleza correlacional de los estadísticos utilizados. La investigación futura debe realizar estudios longitudinales con los que podemos determinar de qué forma el conflicto de rol de género explica el acoso escolar o viceversa. Además, la mayor parte de la investigación revisada y utilizada para discutir los datos obtenidos proviene de otras culturas y contextos sociales diferentes al español, por lo que las comparaciones deben ser consideradas con precaución. También la utilización de escalas de autoinforme, aunque efectivas, están sujetas a la deseabilidad social y a la interpretación de los participantes. En este sentido, sería importante adoptar aproximaciones cualitativas para conocer cómo razonan los jóvenes acerca del conflicto de rol de género.

\section{Referencias bibliográficas}

ARTZ, Sibylle; WASSILIS Kassis y MOLDENHAUER, Stephanie. «Rethinking indirect aggression: the end of the mean girl myth». Victims \& Offenders 8 (2013), pp. 308-328.

BERGER, Jill M. et al. «Impact of Gender Role Conflict, Traditional Masculinity Ideology, Alexithymia, and Age on Men's Attitudes toward Psychological Help Seeking». Psychology of Men \& Masculinity 6 (2005), pp. 73-78.

BlaZINA, Christopher y WatKINS, Edward. «Separation/individuation, parental attachment, and male gender role conflict: Attitudes toward the feminine and 
the fragile masculine self». Psychology of Men $\varepsilon$ Masculinity 1 (2000), pp. 126-132.

BRUCH, Monroe A. et al. "Shyness, masculine ideology, physical attractiveness, and emotional inexpressiveness: Testing a mediational model of men's interpersonal competence». Journal of Counseling Psychology 45 (1998), pp. 84-97.

CARD, Noel A. et al. «Direct and indirect aggression during childhood and adolescence: A meta-analytic review of gender differences, intercorrelations, and relations to maladjustment». Child development 79 (2008), pp. 1185-1229.

COHN, Amy y ZEICHNER, Amos. «Effects of masculine identity and gender role stress on aggression in men». Psychology of Men $\mathcal{E}$ Masculinity 7 (2006), pp. 179-190.

CoHn, Amy M.; ZEICHner, Amos y SEIBERT, Alana. «Labile affect as a risk factor for aggressive behavior in men». Psychology of Men \& Masculinity 9 (2008), pp. 29-39.

COCKER, Ann L. et al. «Dating violence victimization and perpetration rates among high school students». Violence against women 20 (2014), pp. 1220-1238.

GINI, Gianluca y PozzoLI, Tiziana. «The role of masculinity in children's bullying». Sex Roles 54 (2006), pp. 585-588.

Glomb, Stephen M. y EsPelaGE, Dorothy L. «The Influence of Restrictive Emotionality in Men's Emotional Appraisal of Sexual Harassment: A Gender Role Interpretation». Psychology of Men E Masculinity 6 (2005), pp. 240-253.

Good, Glenn E. y MinTz, Laurie B. «Gender role conflict and depression in college men: Evidence for compounded risk». Journal of Counseling E Development 69 (1990), pp. 17-21.

GOOD, Glenn E. et al. «Male gender role conflict: Psychometric issues and relations to psychological distress». Journal of Counseling Psychology 42 (1995), pp. 3-10.

HAYES, Jeffrey A. y MAHALIK, James R. «Gender role conflict and psychological distress in male counseling center clients». Psychology of Men E Masculinity 1 (2000), pp. 116-125.

KASSING, Leslee R.; BeESLEy, Denise y FREY, Lisa. «Gender role conflict, homophobia, age, and education as predictors of male rape myth acceptance». Journal of Mental Health Counseling 27 (2005), pp. 311-328.

LARRAÑAGA, Elisa et al. «Validación de la versión española de la Gender Role Conflict Scale for Adolescents (GRCS-A)». Anales de psicología 29 (2013), pp. 549-557.

JAKUPCAK, Matthew; LISAK, David y ROEMER, Lizabeth. «The role of masculine ideology and masculine gender role stress in men's perpetration of relationship violence». Psychology of Men E Masculinity 3 (2002), pp. 97-106. 
MAHALIK, James R. et al. "A cross-national and cross-sectional comparison of men's gender role conflict and its relationship to social intimacy and selfesteem». Sex Roles 45 (2001), pp.1-14.

Monk, Debra y RiCCIARDELLI, L.A. "Three dimensions of the male gender role as correlates of alcohol and cannabis involvement in young Australian men». Psychology of Men E Masculinity 4 (2003), pp. 57-69.

NAVARro, Raúl; LARRAÑAGa, Elisa y Yubero, Santiago. «Bullying-victimization problems and aggressive tendencies in Spanish secondary schools students: the role of gender stereotypical traits». Social Psychology of Education 14 (2011), pp. 457-473.

NAVARRO, Raúl; LARRAÑAGA, Elisa y YubERO, Santiago. «Gender Identity, GenderTyped Personality Traits and School Bullying: Victims, Bullies and Bully-Victims». Child Indicators Research (2015) pp.1-20.

O'NeIL, James M. «Summarizing 25 years of research on men's gender role conflict using the gender role conflict scale new research paradigms and clinical implications». The Counseling Psychologist 36 (2008), pp. 358-445.

O'NeIL, James M. «Assessing men's gender role conflict». En D. Moore y F. Leafgren (Eds.), Problem solving strategies and interventions for men in conflict, Alexandria, VA, American Association for Counseling, 1990, pp. 23-38.

O’NeIL, James M., et al. «Gender-role conflict scale: College men's fear of femininity». Sex Roles, 14 (1986), pp. 335-350.

RANDO, Robert A.; Rogers, James R. y BRITTAN-POWEll, Christopher S. «Gender role conflict and college men's sexually aggressive attitudes and behavior». Journal of Mental Health Counseling 20 (1998), pp. 359-369.

RigBY, Ken y BAGSHAw, Dale. «Prospects of adolescent students collaborating with teachers in addressing issues of bullying and conflict in schools», Educational Psychology 23 (2003), pp. 535-546.

Rochlen, Aaron B. y MAHAlik, James R. «Women's Perceptions of Male Partners' Gender Role Conflict as Predictors of Psychological Well-Being and Relationship Satisfaction». Psychology of Men E Masculinity 5 (2004), pp. 147-157.

SCHWARTZ, Jonathan P.; WALDO, Michael y DANiEL, David. «Gender-Role Conflict and Self-Esteem: Factors Associated With Partner Abuse in Court-Referred Men». Psychology of Men E Masculinity 6 (2005), pp. 109-113.

Sharpe, Mark J.; HePPNER, Paul y WAYne, Dixon. «Gender role conflict, instrumentality, expressiveness, and well-being in adult men». Sex Roles 33 (1995), pp. 1-18.

THEODORE, Harry y LlOYd, Bibian. «Age and gender role conflict: A cross-sectional study of Australian men». Sex Roles 42 (2000), pp. 1027-1042.

WiTT, Susan D. «The influence of peers on children's socialization to gender roles». Early Child Development and Care 162 (2000), pp. 1-7. 